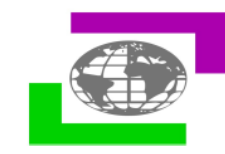

\title{
SURVEY ON ISOLATION TECHNIQUES USED BY ENDODONTISTS AND GENERAL DENTISTS
}

Dr. Vandana James ${ }^{1}$, Dr. Sundaresan Balagopal ${ }^{2}$, Varshinee.G.J. ${ }^{3}$, Dr. Sunil Chandy Varghese ${ }^{4}$, Vaishnavi. ${ }^{5}$, Manishaa.V. ${ }^{6}$

${ }^{1}$ MDS., Reader, Department of Conservative Dentistry and Endodontics, Tagore Dental College and Hospital, Rathinamangalam, Chennai - 600127.

${ }^{2}$ MDS., MSc., Professor and HOD, Department of Conservative Dentistry and Endodontics, Tagore Dental College and Hospital, Rathinamangalam, Chennai - 600127.

${ }^{3}$ CRRI, Tagore Dental College and Hospital, Rathinamangalam, Chennai-600127

${ }^{4}$ MDS., Reader, Department of Orthodontics, Tagore Dental College and Hospital, Rathinamangalam, Chennai - 600127.

${ }^{5}$ CRRI, Tagore Dental College, Rathinamangalam, Chennai-600127

${ }^{6}$ Manishaa.V., CRRI, Tagore Dental College and Hospital, Rathinamangalam, Chennai-600127

Article Info: Received 20 June 2019; Accepted 18 July. 2019

DOI: https://doi.org/10.32553/jbpr.v8i5.643

Corresponding author: Varshinee.G.J

Conflict of interest statement: No conflict of interest

\section{ABSTRACT:}

Aim: To determine the most preferred and used isolation technique by Endodontists and General Dentists during their treatment. To see whether choice of isolation technique differs among Endodontists and General Dentists.

Materials and Methods: A preformed questionnaire was provided to the Endodontists and General Dentists regarding usage of isolation techniques in their practice. Informed consent was obtained from the dentists before answering the questionnaire. STATISTICAL ANALYSIS USED: Analyses were done using SPSS. The main outcomes of interest were the frequency of use of different types of isolation techniques, with attitudes as the key predictors. Scores were used to examine the relationship between the dentist's attitudes about isolation techniques and whether these techniques were used.

Results: The most commonly preferred isolation technique is found to be rubber dam and the choice of isolation technique differed among Endodontists and General Dentists, Endodontists most commonly using rubber dam than General Dentists who preferred using cotton rolls.

Conclusion: From this survey we can conclude that Endodontists mostly prefer using rubber dam due to their increased knowledge about the isolation technique. Knowledge among General Dentists about rubber dam has to be increased.

Keywords: Isolation technique, Rubber dam, Endodontists, General dentists.

\section{INTRODUCTION}

Achieving proper isolation in oral cavity poses a problem by the presence of saliva, micro organism and interference from patient's tongue and soft tissue. Improper isolation affects the dental procedure and its results. ${ }^{[8]}$

Endodontic treatment like other aspects of dentistry, benefits from the use of asepsis. ${ }^{[7]}$ Aerosols and droplets are produced by the use of air turbine that contaminates the field with bacteria and blood. They represent a potential route for transmission of infectious diseases such as measles, tuberculosis, SARS, hepatitis and AIDS [6]

Nonsurgical root canal treatment (RCT) procedures considers rubber dam as the standard of care. ${ }^{[1]}$ When operator convenience and comfort of the patient were considered, the best method remains the use of rubber dam. Though Alternative techniques to rubber dam do exist, use of it always presents as a compromise. ${ }^{[7]}$ Rubber dam application is not a very time consuming process. This brief time spent is more than repaid by proper isolation from patients tongue and lips, salivary contamination and elimination of cotton wool rolls 
and rinsing. The pleasant operating conditions are advantageous. ${ }^{[7]}$

Rubber dam usage results in a significant reduction in the microbial content of air turbine aerosols produced and reduces the risk of cross-infection in the dental practice ${ }^{[6]}$. This is due to its function as a barrier to prevent oral pathogens from entering the tooth and preventing instruments, medicaments from being swallowed or inhaled. ${ }^{[1]}$

Root canal treatment (RCT) is considered as an essential element in the dental services. Total elimination of microorganisms from the root canal system is the goal of endodontic treatment. Successful Root Canal Treatment depends not only on factors like infection, root canal morphology, etc. but is also very much influenced by less specific causes such as dentist's skills and attitudes. [3]

A previous study conducted by the former regional Dental Practice-Based Research Network (DPBRN) determined that $44 \%$ of General dentists (GDs) reported always using an Rubber dam for Root Canal Treatment procedures. The study found that the most common alternative isolation methods were cotton rolls and gauze squares. ${ }^{[1]}$ It showed that Most General dentists do not use Rubber Dam all the time. ${ }^{[1]}$ Rubber Dam usage varies with dentists. Usage of this rubber dam is prevalent among Endodontists. Some General dentists prefer using cotton rolls when compared to rubber dam which offers less protection. Attitudes of these dentists are not known to us.

\section{MATERIALS AND METHODS:}

A total of 90 Endodontists and 90 General dentists were selected.

Under the category of 90 Endodontists they were further divided into the subcategory such as practicing with private clinic, academician and consultation dentists with each subcategory having 30 dentists.

Under the category of 90 General dentists it was divided into the subcategory as given as above ${ }^{[5]}$. Questionnaire was prepared and given to both the Endodontists and General dentists. Before answering the questionnaire informed consent was obtained from the dentists. ${ }^{[1]}$
All Endodontists and General dentists above 25 years of age were included in the study. Nonpracticing dentist were excluded from the study.

Content of the questionnaire for the dentist:

A self administered and a structured questionnaire was given to the dentist. ${ }^{[3]}$ After confirming on the questionnaire that the respondent performs root canal treatment, it was distributed to the dentists. ${ }^{[2]}$ Questions were based on knowledge ,attitude, practices (KAP) study.

A list of 90 general dental practitioners was obtained from the local Indian Dental Association branch. Root canal treatment performing dentists came under the inclusion criteria. Study sample consisted of 90 dentists. To meet the objectives of the survey a total of 90 dentists will be sampled by convenience sampling method (non probability method).

Informed consent was obtained from the participating dentist. Questionnaire was made with 30 questions and it was found to be clear and unambiguous by conducting pilot study among 20 dentists. ${ }^{[3]}$ Investigator accompanied the dentist while the questionnaire was been answered. The questions consisted of statements regarding the use of isolation technique and the reason for its usage. The questions also assessed the attitudes of the dentists regarding the isolation technique and this was used as a predictor for assessment. Questions were asked regarding the usage of rubber dam, effectiveness of rubber dam, potential problems of rubber dam usage.$^{[2]}$ Questions were also given regarding the usage of cotton rolls. Differing choices of isolation techniques among dentists was assessed.

The questionnaire involved information on the respondent's individual characteristics such as his/her Job history, goals of isolation and most commonly used isolation technique. The independent variables in the questionnaire will be about whether he/she is a academician or a consultant or a private practitioner.

The questionnaire consisted of questions regarding the choice of isolation technique for various procedures in dentistry such as root canal treatment, restorative procedures. Differing choices of isolation technique among consultants, private clinicians and academicians was assessed. 
The preference of one technique over the other one was assessed and the reason for the difference in usage was found.

Analyses were done using SPSS. The main outcomes of interest were the frequency of use of different types of isolation techniques, with attitudes as the key predictors. Scores were used to examine the relationship between the dentist's attitudes about isolation techniques and whether these techniques were used. ${ }^{[2]}$

\section{RESULTS:}

Details on eligibility, response rates, differences between participants and non-participants, and characteristics of participants have been previously reported. The purpose of this paper is to review the different aspects of rubber dam use in root canal treatment and discuss the possibilities to popularize its use amongst dental practitioners. ${ }^{[6]}$

Most commonly used isolation technique:

This survey reveals that most commonly used isolation technique among Endodontists is found to be rubber dam and the most commonly used technique by General dentists is found to be cotton rolls.

Efficacy of different isolation techniques:

Even though the usage of the most efficacious rubber dam technique was found to be low, both General dentists and Endodontists agreed that rubber dam is the most efficient isolation technique.

Isolation technique usage differences among Endodontists and General dentists:

This survey reveals that among Endodontists academician, consultants and private clinicians mostly prefer using rubber dam when compared to other techniques such as cotton rolls and saliva ejectors.

General dentists due to lack of practice and knowledge most commonly use saliva ejectors and cotton rolls when compared to rubber dam.
Difficulty in placement and time involved in placing the rubber dam has been seen as the reason for limited use of rubber dam.

Compared with the dentists, students took longer to apply rubber dam and it was in place for longer. Fewer student patients preferred RubberDam next time, and were less positive about its use than the dentists' patients. ${ }^{[5]}$ Surveys undertaken in several countries reported rates of rubber dam usage when performing endodontic treatment There is a discrepancy between the frequency of rubber dam use between undergraduate students and practicing dentists. ${ }^{[6]}$

\section{choice of isolation technique}

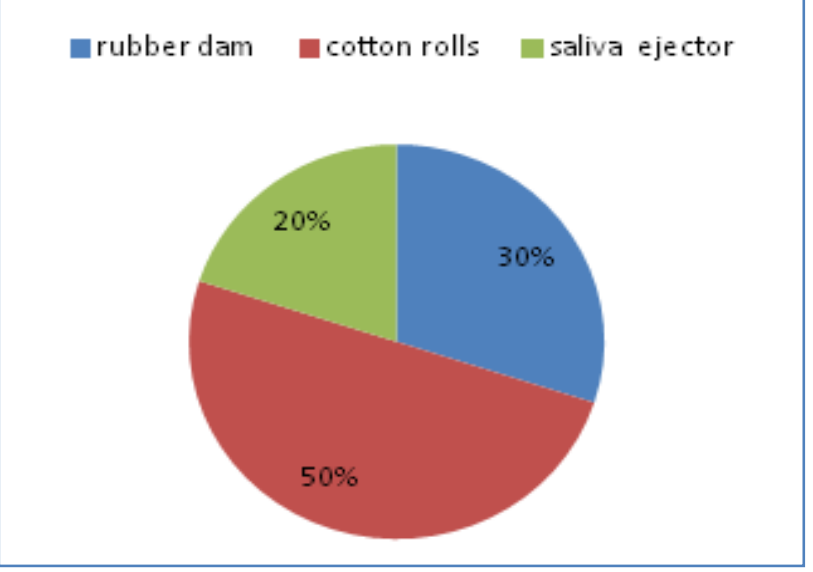

Figure 1:

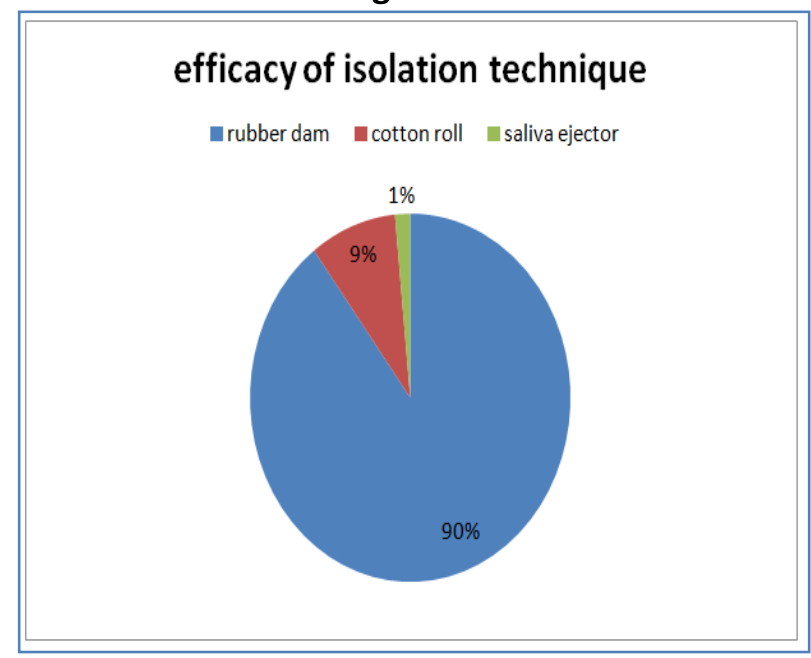

Figure 2: 
Varshinee.G.J et al., Journal of Biomedical and Pharmaceutical Research

Table 1: DIFFERENCE IN USAGE OF ISOLATION TECHNIQUE AMONG DENTISTS:

\begin{tabular}{|l|l|l|l|l|l|l|}
\hline $\begin{array}{l}\text { ISOLATION } \\
\text { TECHNIQUE }\end{array}$ & \multicolumn{2}{l|}{ ENDODONTISTS-90 } & \multicolumn{2}{l|}{ GENERAL DENTISTS-90 } \\
\hline $\begin{array}{l}\text { CONSULTANT } \\
30\end{array}$ & $\begin{array}{l}\text { ACADEMICIAN } \\
30\end{array}$ & $\begin{array}{l}\text { PRIVATE } \\
\text { CLINICIAN } \\
30\end{array}$ & $\begin{array}{l}\text { CONSULTANT } \\
30\end{array}$ & $\begin{array}{l}\text { ACADEMICIAN } \\
30\end{array}$ & $\begin{array}{l}\text { PRIVATE } \\
\text { CLINICIAN } \\
30\end{array}$ \\
\hline RUBBER DAM & 15 & 25 & 20 & 5 & 15 & 10 \\
\hline COTTON ROLLS & 10 & 4 & 5 & 15 & 10 & 10 \\
\hline $\begin{array}{l}\text { SALIVA } \\
\text { EJECTOR }\end{array}$ & 5 & 1 & 5 & 10 & 5 & 10 \\
\hline
\end{tabular}

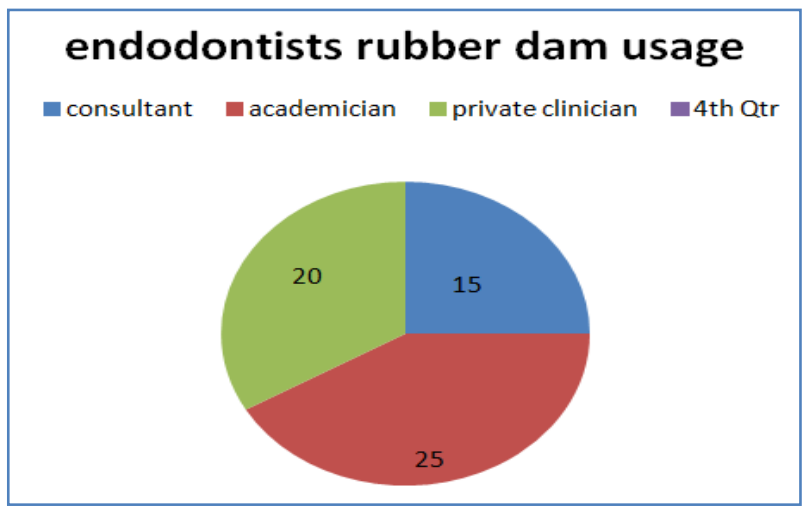

Figure 3:

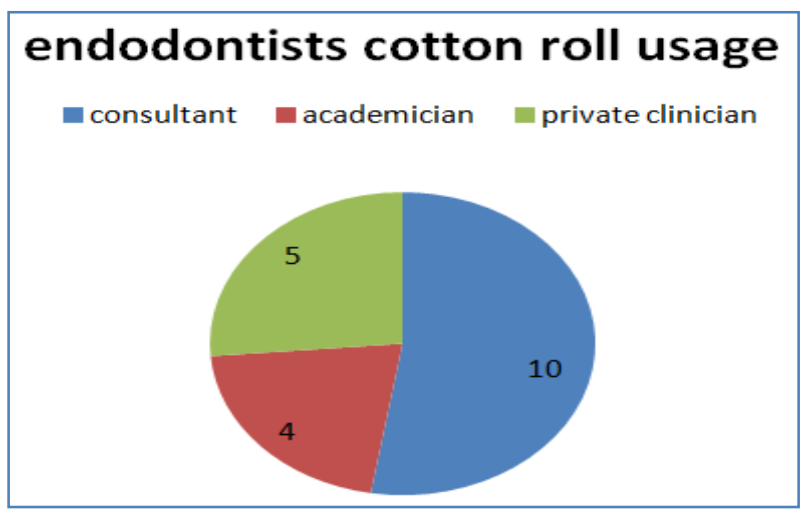

Figure 4:

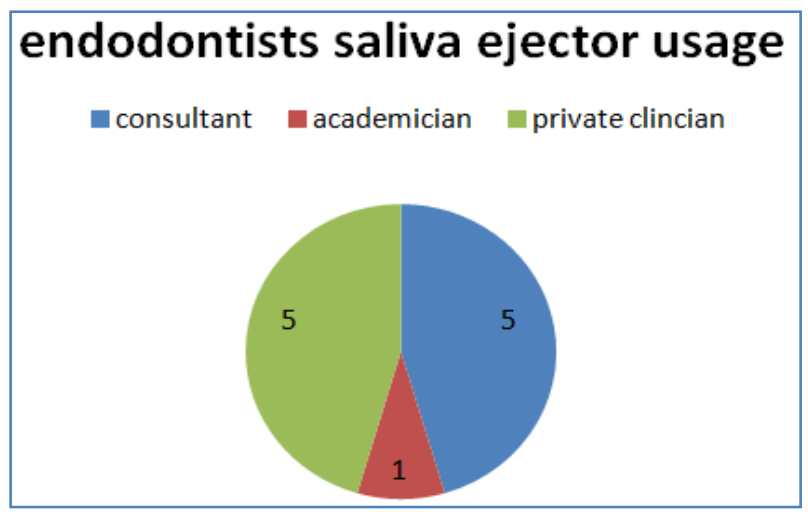

Figure 5:

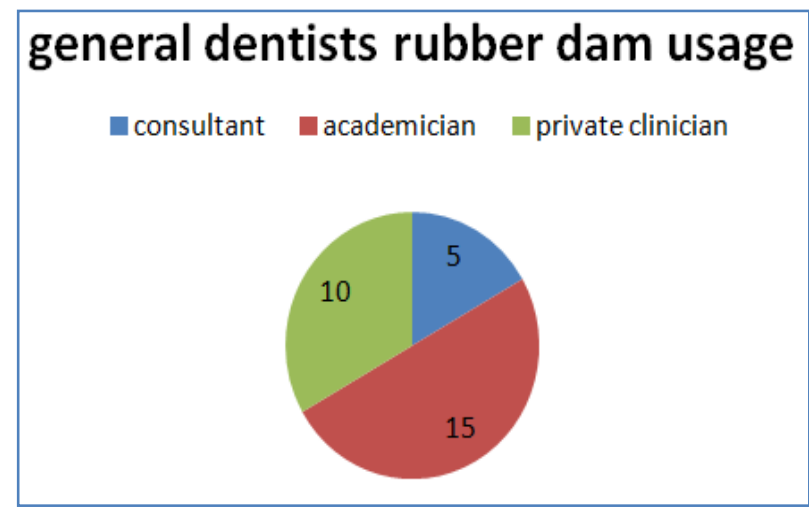

Figure 6:

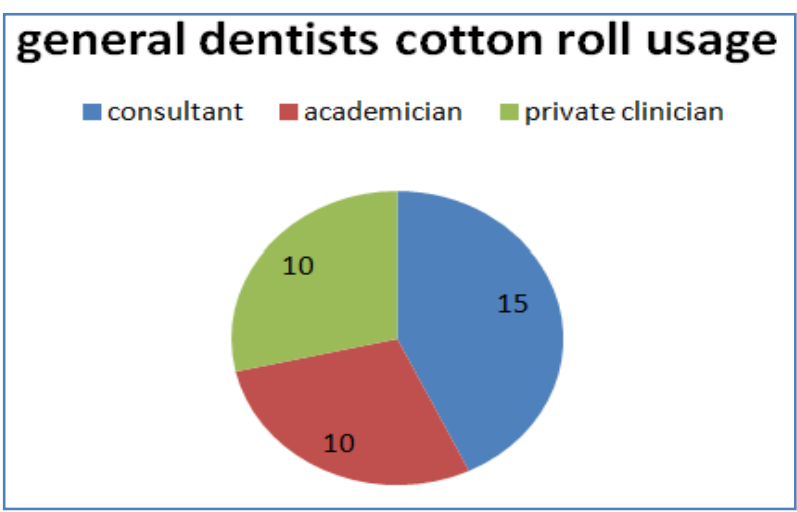

Figure 7:

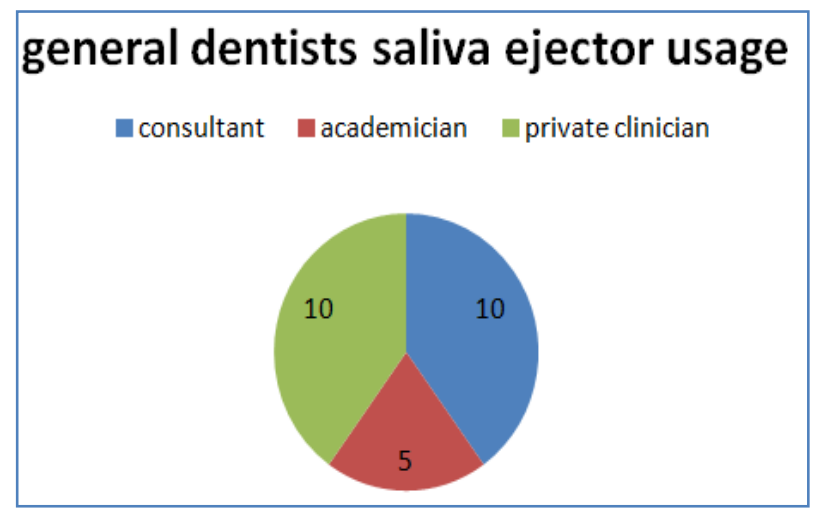

Figure 8: 


\section{DISCUSSION:}

The concept of absolute isolation was developed more than 140 years ago. Despite this its usage has been less. it has widely acknowledged benefits and even though a number of clinicians refuse to use absolute isolation in routine endodontic clinical practice $^{[8]}$.With a view to improving clinicians' and patients' conditions, the absolute isolation technique has been modified over time.

In spite of the research about rubber dam, one question remains un answered: why a universally advocated technique is not practiced by the majority of working dentists? Some investigators demonstrated the dental techniques taught in the dental school, including rubber dam, are not consistently applied once the graduates settle into clinical practice. ${ }^{[6]}$

In UK, only about a quarter of respondents routinely used rubber dam during root canal therapy; however, in America 59\% and in New Zealand $57 \%$ of the dentists were using rubber dam routinely in endodontic treatment. In a similar study, conducted in KSA, only $3 \%$ of the respondents were using rubber dam as the method of isolation. This lack of use of rubber dam can directly affect the standard of root canal treatment and decrease the success rate. ${ }^{[10]}$ Many studies done over the world reveal that general dentists do not use rubber dam. ${ }^{[11]}$

\section{General dentists:}

In this study we determined that most of the General dentists do not use rubber dam when compared to the Endodontists. The reason for this includes patient discomfort, time required for placement, difficulty in use, and cost of equipment. ${ }^{[1]}$ The finding is such that General Dentists who have less specialist interaction are less influenced to use an RubberDam. General dentists who had additional training had higher compliance with Rubber Dam usage. These findings suggest that education, particularly related to the benefits of Rubber Dam usage, is more effective in clinical setup. ${ }^{[1]}$

Adequate isolation with a cotton roll alone is very challenging in the oral cavity because of the salivary ducts, gravitational pooling of saliva, and insufficient retention of the cotton roll. Some clinicians use cotton rolls as a supplemental form of isolation but many dentists use them as the main form of isolation. Several of these clinicians included comments that continuous high-volume saliva ejection was used to supplement the use of cotton rolls. Dentists who learned endodontic techniques in general practice residency training or continuing education courses may be more likely to adhere to recommendations for Rubber Dam use. [1]

\section{Endodontists:}

Rubber dam enables a higher clinical standard to be achieved. From the results we conclude that several key factors are associated with rubber dam use. Attitudes about rubber dam use for treatment effectiveness and patient safety, seem to be the main factors, with additional factors such as inconvenience, ease of usage, and patient considerations ${ }^{[2]}$.certain effects could affect the results such as additional training and experience which affects the attitudes of the dentists. ${ }^{[2]}$ From this survey we can conclude that Endodontists mostly prefer using rubber dam due to their increased knowledge about the isolation technique.

The results showed that frequent users were significantly less likely to cite the reasons (except for the cost of materials) than nonusers.

With a view to improving clinicians' and patients' conditions, the absolute isolation techniques have been modified over time.

\section{REFERENCES:}

1. General Dentists' Use of Isolation Techniques during Root Canal Treatment: From the National Dental Practice-based Research Network Nathaniel C. Lawson, DMD, PhD, Gregg H. Gilbert, DDS, MBA, Ellen Funkhouser, Dr PH, Paul D. Eleazer, DDS, MS, Paul L. Benjamin, DDS, and Donald C. Worley, DDS, NATIONAL DENTAL PBRN COLLABORATIVE GROUPvol no: , issue no: , pages:1-7, 2005

2. Discordance between presumed standard of care and actual clinical practice: the example of rubber dam use during root canal treatment in the National Dental Practice-Based Research Network Gregg H Gilbert,1 Joseph L Riley,2 Paul D Eleazer,3Paul L Benjamin,4Ellen Funkhouser,5 National Dental PBRN Collaborative Group- vol no: ,issue no: , pages:1-7, 2015

3. Attitude of General Dental Practitioners toward Root Canal Treatment Procedures in IndiaAshwini Gaikwad, Deepak Jain, Prasad Rane, Sarvesha 
Varshinee.G.J et al., Journal of Biomedical and Pharmaceutical Research

Bhondwe, Swapnil Taur, Saurabh Doshi- vol no: 14 ,issue no: 3 , pages:528-531,2013;

4. Patients' attitudes to rubber dam. Stewardson DA1, McHugh ES. vol no35: ,issue no:10, pages:812-9, 2002

5. Prevalence of Rubber Dam Usage during Endodontic Procedure: A Questionnaire Survey.G S1, Jena A1, Maity AB2, Panda PK3. 2014 Jun; vol no:8(6): pages:1-3

6. Rubber dam usage for endodontic treatment: a review I. A. Ahmad Private Dental Practice, Amman, Jordan vol no: 42 ,issue no: , pages:963972,2009;

7. The isolation of teeth and the protection of the patient during endodontic treatment, j.e.reuter vol no: 14 , issue no: 3 , pages:528-531,2013;

8. A new option for complete isolation in endodontics- RESEARCH GATE Sérgio Henrique Staut BRUNINI1 Renan Lucas da Silva PIMENTA2
Lucas Rafael Rodriguez de SOUZA vol no: 4 ,issue no: 2, pages:83-7,2014;

9. The Experience and Attitude of Saudi Patients towards Rubber Dam Isolation during Dental Treatments Basil Yousif Alamassi1, Mowaffq Shafi2, Ahmed Alenezi2, Abdullah Alghamdi2, Mohammed Alerredi2,Omar Alwazzan2 =IOSR Journal of Dental and Medical Sciences (IOSRJDMS) Volume 16, Issue 4 Ver. I (April. 2017), PP 70-7;

10. A Survey of Standard Protocols for Endodontic Treatment in North of KSAAzharlqbal,1 IftikharAkbar,1 BeenishQureshi,2 MohdG. Sghaireen, 3 andMahmoudK.AL-Omiri4,5 Volume 2014, Article ID 865780, 4 pages;

11. Rubber dam placement why majority of senior dentists practicing in Saudi Arabia ignore it? Vol20, issue- 1, January 2017 